\title{
A New Cultural Lens to Unpack Idiosyncratic Business Behaviors: An Abstract
}

\author{
Xin Yang
}

\begin{abstract}
Cultural ascription orientation refers to people's cultural tendency of using ascribed attributes to evaluate others and their social status. Whereas previous literature commonly conceptualizes ascription orientation as a mirror of achievement orientation along the same cultural dimension, two empirical studies are presented to demonstrate the uniqueness of cultural ascription orientation as an independent dimension to account for the variations of business behaviors across cultures. Findings from secondary data on 41 nations demonstrated that cultural ascription orientation is a unique dimension (Study 1), distinct from the conventional cultural dimensions proposed in Hofstede's (1980) study. Study 2, used cultural priming techniques in an experiment, testified that consumers primed with ascription-oriented values have a different attitude toward the VIP status of the company than customers primed with achievement-oriented values. Together, the above two studies reveal that cultural ascription orientation possesses unique explanatory power to account for the idiosyncratic business behaviors of people across nations.
\end{abstract}

References Available Upon Request

\footnotetext{
X. Yang $(\bowtie)$

Hang Seng Management College, Sha Tin, Hong Kong

e-mail: morganyang@hsmc.edu.hk
} 